Original Research Paper

\title{
A Scheme Design of Cloud + End Technology in Demand Side Management
}

\author{
${ }^{1}$ Leijiao Ge and ${ }^{2}$ Xiandong Xu \\ ${ }^{I}$ School of Electrical Engineering and Automation, Tianjin University, Tianjin, China \\ ${ }^{2}$ School of Electronics, Electrical Engineering and Computer Science, Queen's University Belfast, Belfast 95 AH, UK
}

Article history

Received: 26-09-2015

Revised: 16-10-2015

Accepted: 02-11-2015

Corresponding Author:

Xiandong $\mathrm{Xu}$

School of Electronics, Electrical

Engineering and Computer

Science, Queen's University

Belfast, Belfast $95 \mathrm{AH}$, UK

Email: x.xu@qub.ac.uk

\begin{abstract}
Demand Side Management (DSM) plays an important role in Smart Grid. It has large scale access points, massive users, heterogeneous infrastructure and dispersive participants. Moreover, cloud computing which is a service model is characterized by resource on-demand, high reliability and large scale integration and so on and the game theory is a useful tool to the dynamic economic phenomena. In this study, a scheme design of cloud + end technology is proposed to solve technical and economic problems of the DSM. The architecture of cloud + end is designed to solve technical problems in the DSM. In particular, a construct model of cloud + end is presented to solve economic problems in the DSM based on game theories. The proposed method is tested on a DSM cloud + end public service system construction in a city of southern China. The results demonstrate the feasibility of these integrated solutions which can provide a reference for the popularization and application of the DSM in china.
\end{abstract}

Keywords: Cloud Computing, Demand Side Management, Construction Model, Service Platform, Game Theory

\section{Introduction}

Demand Side Management (DSM) is attracting more and more concerns and facing many opportunities and challenges. Technologies such as internet of things, smart grid and smart city, are incorporated into the DSM, which provide a strong implementation conditions for controlling adjustable variables in the extensive user side. Specifically, it includes Distributed Generations (DGs), Electric Vehicles (EVs), Flexible loads and so on. However, for practical applications, there are still a large number of problems needed to be solved including both technique and economic aspects. From the view of technologies, it includes how to solve and analyze the demand response of large-scale users quickly, how to response different users in real time, how to forecast the demand of customers with complex variables and uncertain conditions. Therefore, cloud computing provides a better solution for this problem, which is characterized by super large scale, high reliability and resource on-demand, extremely cheap and so on. From the point of economics, the main problem is how to balance and distribute the investigation and repayment of the DSM among government, the power grid enterprise, energy-saving service company and customers. So, a game theory is a good way to solve these problems.

In the past few decades, a number of studies have investigated DSM including the optimization (Alireza et al., 2014; Logenthiran et al., 2012), model (Malik, 2001; Wang et al., 2014), smart home (Adika and Wang, 2014), electric vehicles (Lopez et al., 2015) and so on.

Moreover, Cloud computing is a useful tool in fields including power system, education, etc[].. In (González-Martínez et al., 2015; Sheikhi et al., 2015), the definition of cloud computing was introduced. The approaches and environments for carrying out analytics on clouds for big data application were discussed in (Assunção et al., 2015). On the other hand, cloud computing is widely discussed including the framework (Sheikhi et al., 2015), virtual machine (Blake, 2007), big data (Assunção et al., 2015).

A game theory is presented to gain deeper insight on the issue of economic in the DSM. Many researchers focus on the game theory, including strategies (Ji et al., 2015), monotony of probability function (Vasile et al., 2012), punishment fraction (Li et al., 2015). 
The organization of this paper is as follows. In section 2, the advantage of cloud computing in the DSM is introduced. In section 3, the technical framework of cloud + end is proposed. Section 4 proposes the game theory method to solve the construction model of the DSM. In section 5, the case verifies the advantage of the cloud + end solution. The paper is concluded in section "Conclusion".

\section{Cloud Computing Technology}

Cloud computing technology is a highly fusion of virtualization and grid computing. Unified management of sever and memory through virtualization is implemented by real-time monitoring and dispatching. IT resource is integrated by grid computing. According to the service mode, cloud computing technology includes three levels: Infrastructure as a Service (IasS), Platform as a Service (PasS) and Software as a Service (SasS). Different from supercomputers, it has strong expansibility and can upgrade its computing capability conveniently. Compared with traditional computing modes, it's easy to integrate and analyze information for system development and maintenance. Furthermore, it also has a low price.

Cloud computing proposed by Google in 2007 has been applied extensively in three forms, private cloud, public cloud and hybrid cloud. Moreover, cloud computing service has been provided by many corporations, such as, Google App Engine, Elastic Compute Cloud, Microsoft's Azure Service Platform, Ali cloud, Tencent cloud and so on.

According to the characteristics of cloud computing technologies, applying cloud computing into the DSM has the following advantages:

- Cloud computing can integrate IT resource and manage data resource through the virtualization, so that the ability of integration can be promoted for serving the mass user in DSM. It can provide a basic computational platform for the DSM

- Cloud computing has superior data processing ability and memory ability and it can greatly promote the DSM's data collection ability and its data processing ability. It's also a lifting for smart grid

- Cloud computing has large-scale management ability. Cloud platform can manage all the intraregion equipment in the DSM center and improve the management level and efficiency. It can also integrate intra-region data information

- Cloud computing has flexible application. The resource utilization can be maintained in a high level by rational utilization of system resources and we can also save equipment investment and reduce manual operation by combining inherent resources and present modes

\section{Technical Framework}

The DSM has a wide range of participants and heterogeneous of hardware and software and involves some hardware and system, such as front-end equipment (smart meter, collector, intelligent terminal, etc.), IT equipment (mobile phone, iPad, etc.), user system platform (home energy management system, micro-grid energy management system, etc.) and so on. It also has many applications in smart distribution system, for example, virtualization of dispersed computers, data storage and resource assignment, as well as fast construction of platform. The business mainly involves multi-level interconnection of energy management among residents, buildings, enterprises, community and smart distribution system. It can achieve the energy saving, emission reduction and efficient utilization of energy in local areas.

Hereby, as shown in Fig. 1, a technical framework of the cloud + end solution in the DSM is proposed, considering the cloud computing technology's analysis and processing ability. According to the idea of hierarchical division management, it consists of a cloud and $\mathrm{N}$ ends.

\section{Cloud}

The cloud is a top decision-makers and the actual controller in the DSM. It is supported by the smart distribution system based on the existing hardware and software in power grid enterprise. It not only manages the business of DSM, but also is responsible for the operation and maintenance of the system. It can be divided into five aspects including infrastructure layer, platform layer, application layer, user layer and cloud security. It is shown in Fig. 2.

\section{Infrastructure Layer}

Corresponding to the cloud service mode's IasS, infrastructure layer is used for unified virtual management of computer resource and data in the DSM and it can be divided into physical layer, data layer and system layer. Among them, physical layer manages server, disk array and PC through virtualization and make router, collector and switch become virtual. Data layer manages big data in cloud by the GHFS technology, MapReduce technology and multi-agent data processing technology. System layer is the control center of basic equipment layer, which is critical in the normal operation. Its components are shown in Fig. 3. 


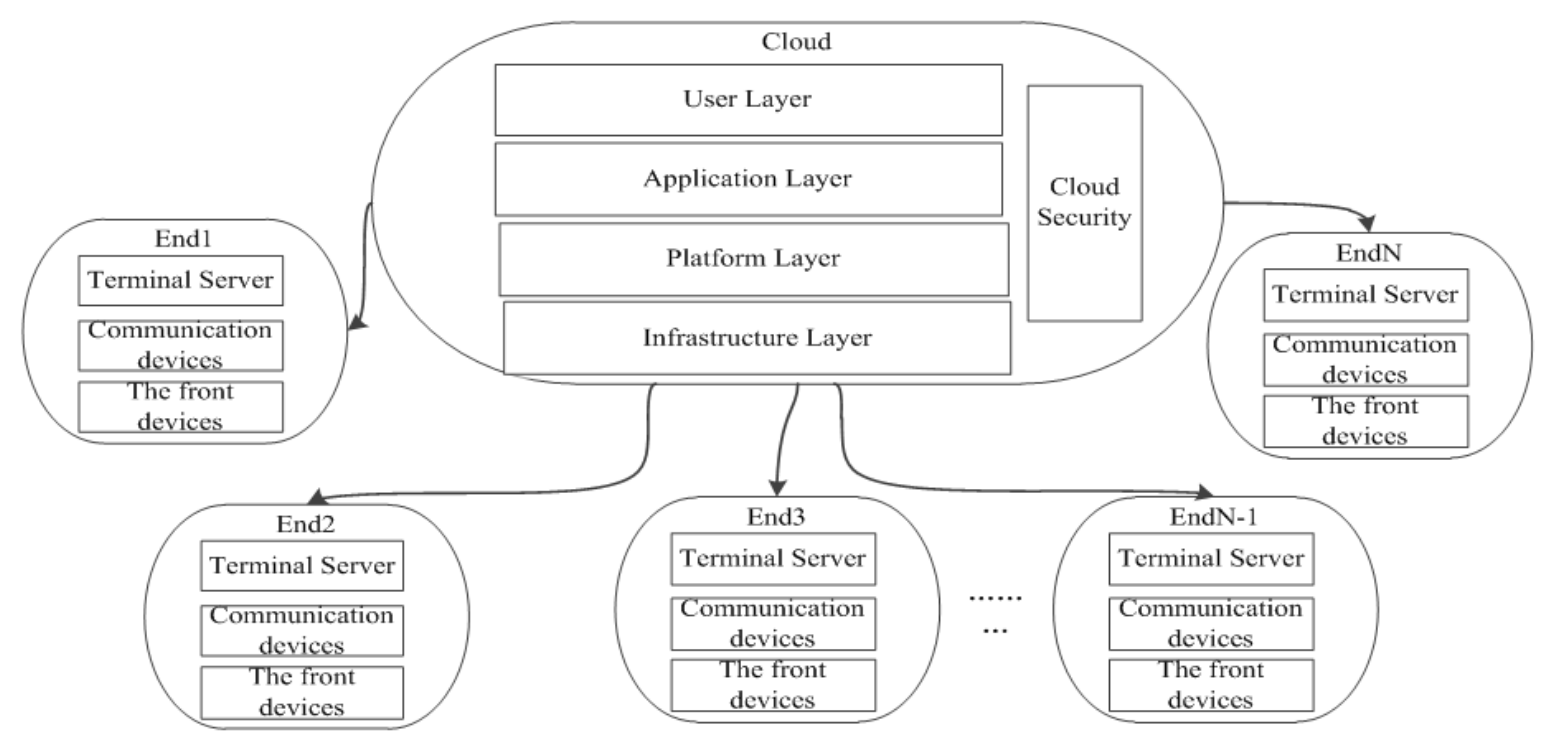

Fig. 1. DSM framework of cloud computing technology

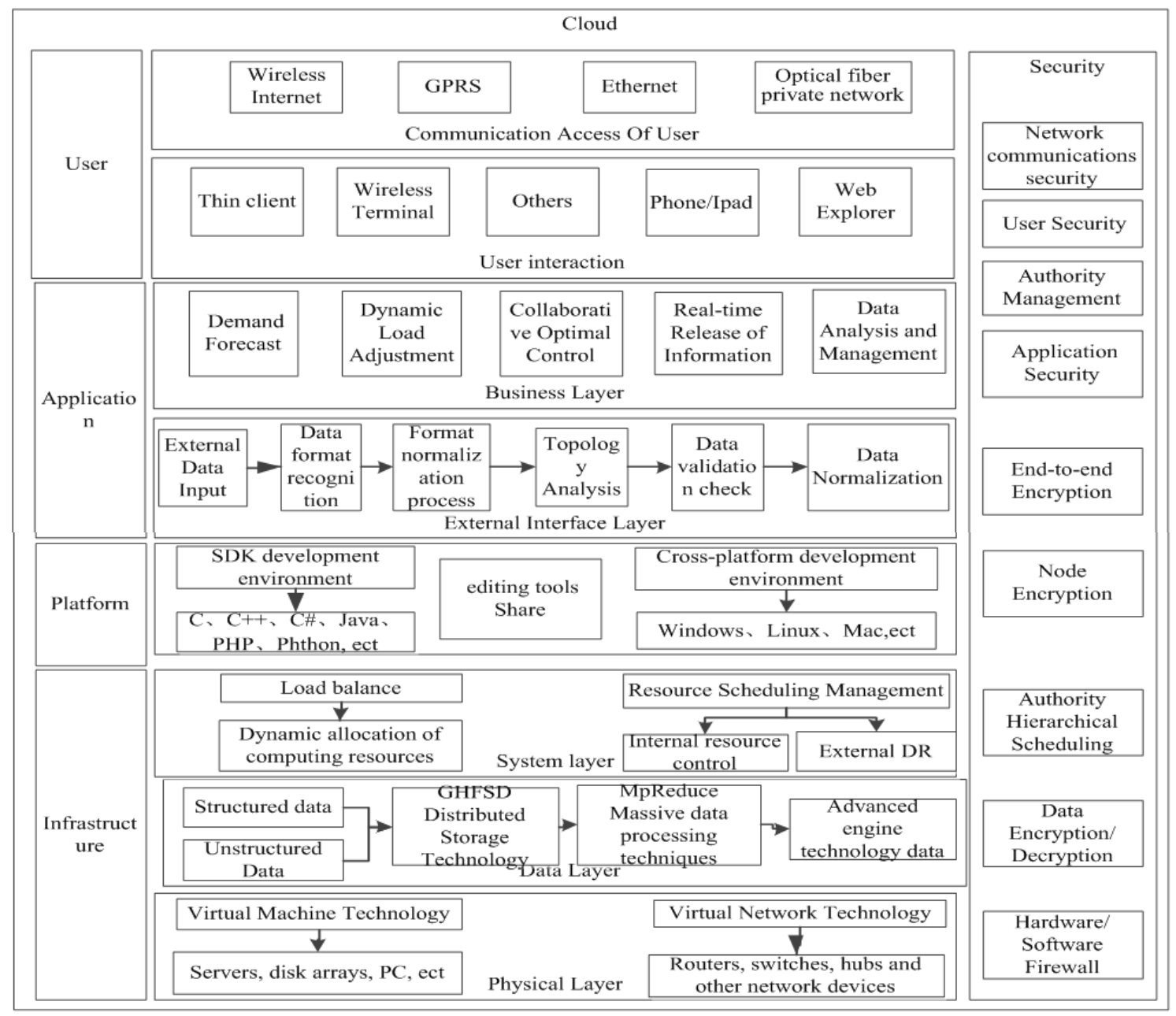

Fig. 2. Cloud framework of DSM 


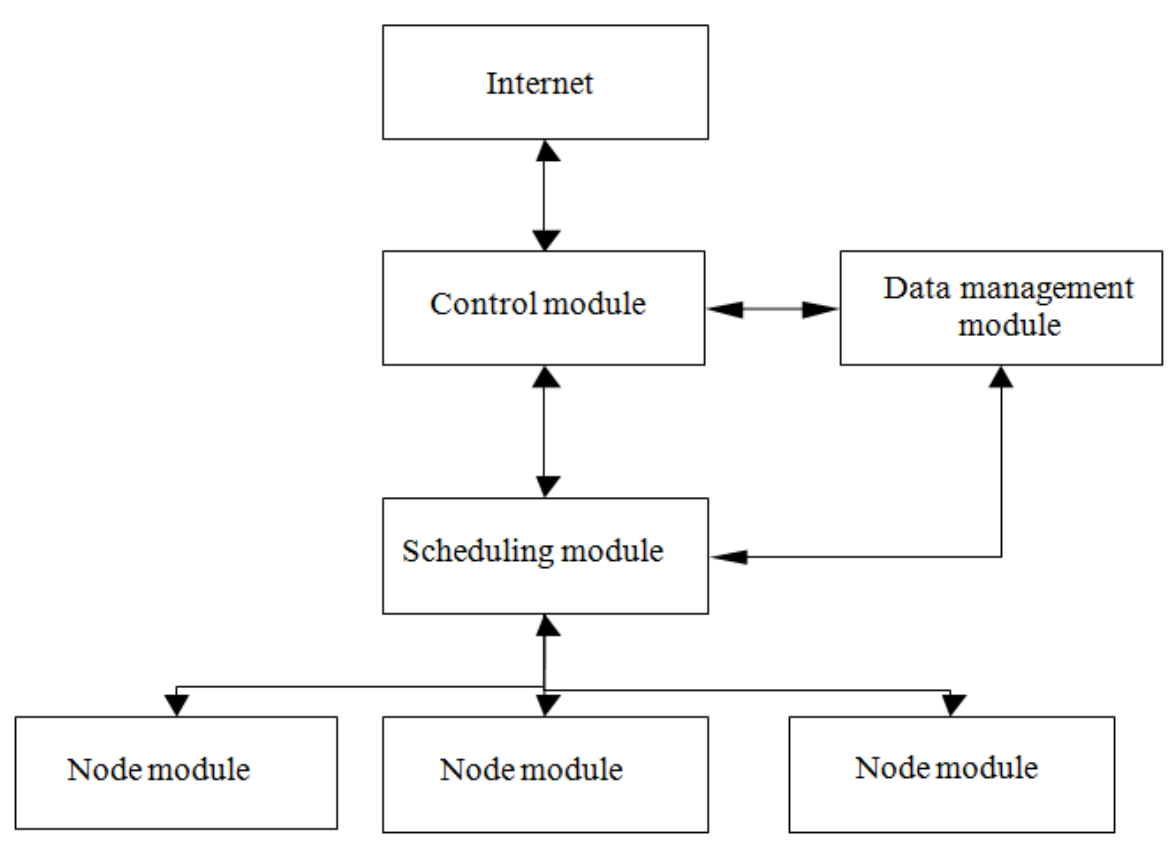

Fig. 3. Function module of the system layer

Table 1. The function module of the system layer

\begin{tabular}{ll}
\hline Module name & Function description \\
\hline Control module & (1) Manage all the computing resources and host computers \\
& (2) Provide platform management for administrator \\
Scheduling module & Manage and monitor the operation of cloud platform, accept users' request \\
Data management module & Manage demand data when platform operate \\
Node module & Operate control module's request and communicate with normal computing node \\
\hline
\end{tabular}

The principle of the work is as follows:

- The control module monitors real-time status of the system and schedules the entire system. It not only exchanges the information with the internet, but also is responsible for the real-time data interaction with the data management module. The scheduling module is managed according to the requirements of the tasks

- The scheduling module is firstly responsive to the control module and then sends the control information to the node module (cloud node). Next, the relevant interactive information is sent to the data management module

- The data management module manages all information resource infrastructure of the cloud. The role of each component is shown in Table 1

\section{Platform Layer}

Corresponding to the PasS, the platform layer provides platform for the development of the DSM and it is a tool library for software developers. It includes three parts as follows:
- SDK development environment that supports the mainstream development language, such as $\mathrm{C}, \mathrm{C}++$, C\#, Java, Python and so on

- Edit tool that can support the mainstream editor

- Cross-platform development environment that has a favorable compatibility of the mainstream operating systems, such as Windows, Linux and Mac, etc

\section{Application Layer}

Corresponding to the SasS, the application layer is the central part of the DSM. It mainly includes external interface and business application. Among them, the external interface is for the data standardization of the DSM's business application. It is a conventional data standardization process including data input, format recognition, normalization processing, topological analysis, validation, standardization and other parts.

As shown in Fig. 4, business application has five sub-modules. The first function is data analysis and management. Then it implements four goals by four different means. The four goals are related to technical methods, economic methods, guide methods and administrative methods and the four means are 
electricity demand forecast, dynamic load adjustment, collaborative optimal control and real-time information release.

\section{User Layer}

User layer is the user interface in the cloud platform. It includes user interactive mode selection and communication mode of user interface. User interactive mode includes client, wireless terminal, phone, iPad, browser and other forms. Communication mode mainly includes internet, GPRS, Ethernet and fiber and communication protocol for main users.

\section{Cloud Security}

Cloud security is an important part in cloud computing technology. Many clients will be used in anomaly detection, auto-analysis and auto-processing. Therefore, the cloud platform will be an anti-virus software. It includes firewall, data encryption, node encryption, application security, authority stratified scheduling and so on.

\section{End}

End is the front part of end framework in DSM. It is used to implement the standardization of equipment access and to correct execution in the DSM. It is composed of the front devices, communication equipment and terminal server, as shown in Fig. 5.

\section{The Front Device}

In order to effectively attribute mass users in demand side, end users are divided into three categories including residents, business and industries. There exists different type of front devices in these end users. The front device involves IT equipment, such as mobile terminals, mobile phones, iPad, Internet and so on and system equipment, for example, Home Energy Management System (EMS), Distribution Supervisory Control and Data Acquisition, Micro-Grid EMS, Building EMS, Factory EMS, etc[]. and terminal equipment, such as intelligent terminals, collectors, concentrators, smart meters and so on.

\section{The Communication Equipment}

The communication equipment is used to support optical fiber network, Ethernet, GPRS, CDMA, PTSN, RS422, RS485, Industry Bus and so on. It installs router management software in each communication device to ensure the completeness of the communication from the terminal server to the front devices. Also, it is responsible for the communication management.

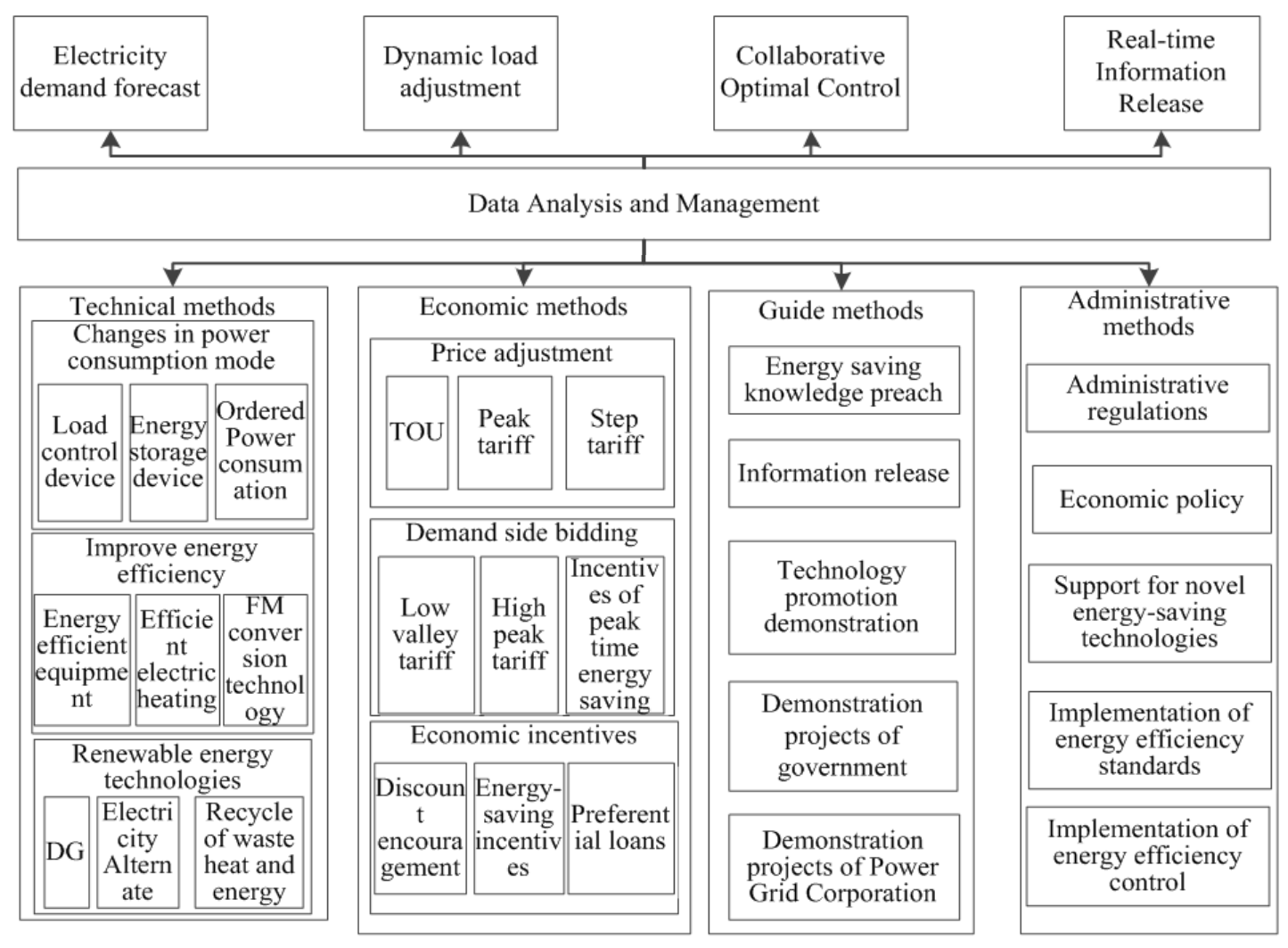

Fig. 4. Structure diagram of DSM business application 


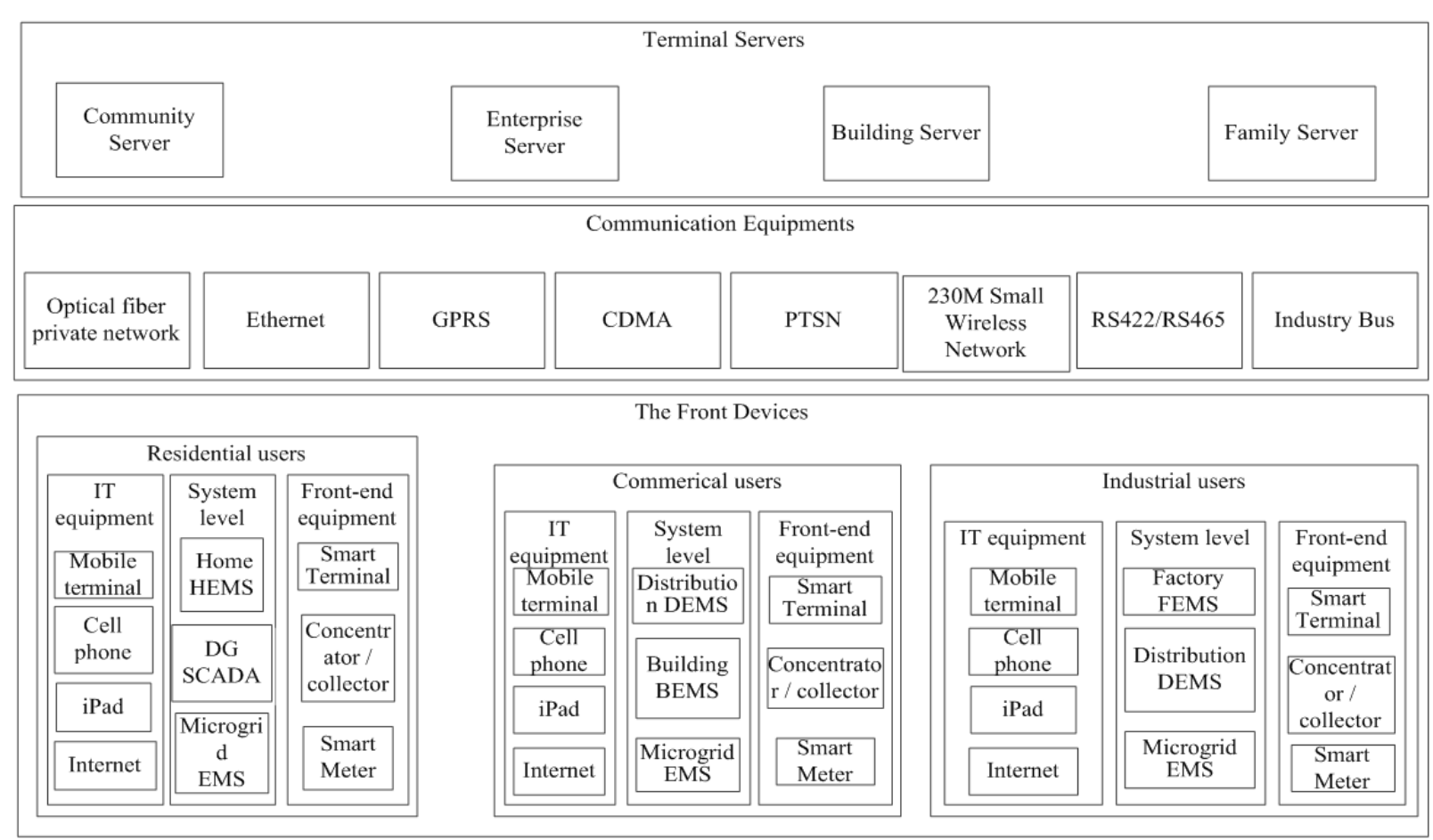

Fig. 5. End framework of DSM

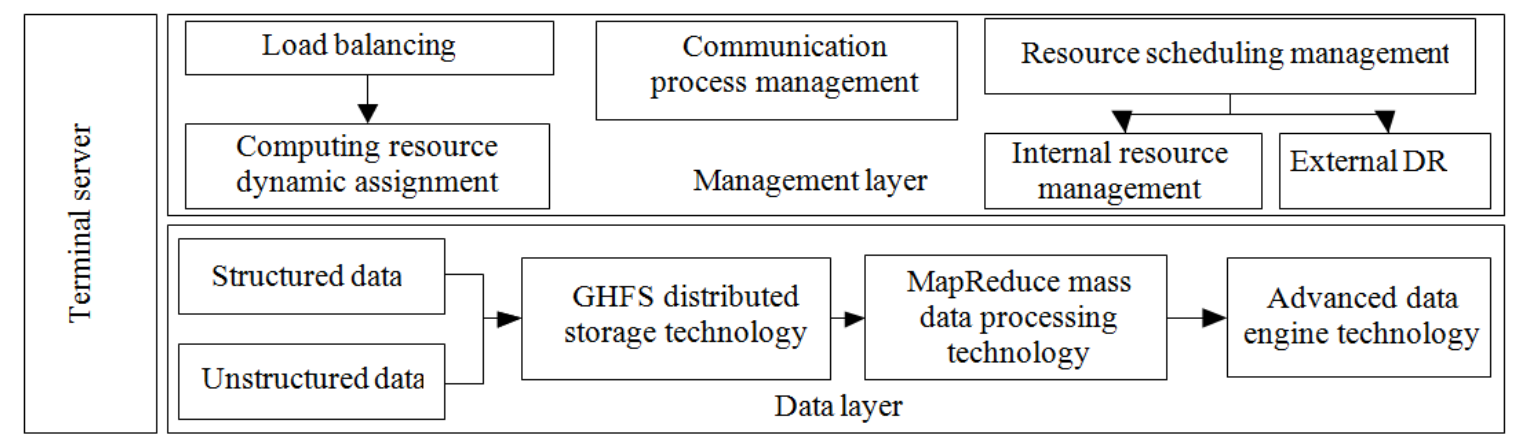

Fig. 6. System framework diagram of terminal server

\section{Terminal Server}

The terminal server is a core in the end layer. It is a supporting platform for the core software of end layer. It is also a hinge to the cloud + end integrated solution. In order to effectively management mass servers in demand side, terminal servers are divided into four categories including community, enterprises, buildings and families.

Terminal server is responsible for the standardization of equipment access in the end layer. On the one hand, it supports many kinds of communication mode and protocol, so that terminal devices can access to the network. On the other hand, there will be a two-way interaction between end and cloud through the terminal server. It can be divided into to two parts from logic including data layer and management layer. System framework of the terminal server is shown in Fig. 6.

\section{Data Layer}

Data layer is in charge of data processing, data storage and data engine. It uses the GHFS technology to memory data based on the data structure. Data layer applies MapReduce technology to data processing. It also uses agent data processing technology to manage data searching.

\section{Management Layer}

Management layer is responsible for dynamic management of data in the terminal layer. It mainly 
includes three processes: Load balancing, communication process management and resource scheduling management. Load balancing is used to implement resource dynamic assignment. Communication process management is used for automatic-selection of communication method. Resource management is used for internal resource scheduling management and external demand response.

\section{Router Optimization Strategy}

The DSM integrated solutions consist of cloud and end. It builds a complex network through the multitype network equipment, connecting the large scale hardware and software in power grid and user side. Moreover, the complex network which is large scale, multi node, many routing cause some problems in the control decision-making process of the DSM, including the network communication congestion, delay, routing and other issues, which greatly affect the actual application effect of the DSM. In some scenarios, the correct scheduling instructions are not reached. When user-side response is changed, it is necessary to recalculate and send scheduling instructions. Furthermore, to avoid this kind of problem, this paper proposes a routing optimization strategy based on non-vector graph theory, which ensures the fast and reliable data transmission between cloud layer and end layer.

\section{Model}

The integrated solution in the DSM is a complex network model. It is composed of a finite number of nodes through a certain physical connection way. As a result, this model can be described as a simplified nonvector graph $G=(V, E)$, which has a weight $\mathrm{W}$, as shown in Fig. 7.

$$
G=(V, E)
$$

These variables of $G(E, V)$ are as follows:

- $\quad V$ is a poor non-empty set of DSM complex network, which is composed of all nodes of the integrated solutions in DSM. A unique set of continuous natural numbers are numbered from 1 to $\mathrm{N}$ for this nodes

- $E$ is a set and indicates the relationship of any two nodes in set $V$. The disorder $(i, j)$ is a boundary value between node $i$ and $j$, represented by $e_{i j}$. The implication of $e_{i j}$ is the transmission time of data packets, which are sent from the node $i$ to node $j$

- In $G=(V, E)$, each $e_{i j}$ of the set $\mathrm{E}$ has only mapping value, which is a positive real number $\mathrm{W}\left(e_{i j}\right)$, then the set $\mathrm{W}\left\{e_{11}, e_{12}, \ldots, e_{n n}\right\}$ is the weight of the novector graph $G$ :

$W_{i j}=T_{1}^{*} n+T_{2}+T_{3}$

where, $W_{i j}$ represents the total time from the node $i$ to node $j$. Similarly, $T_{1}$ is the data packet delivery time, $n$ is node number, $T_{2}$ is blocking time and $T_{3}$ is delay.

By finding the minimum weight of the non-vector graph $G$, it is easy to find that the route optimization strategy is a nonlinear and multi constraint problem. So, in the scheme design of cloud + end technology, there are different heuristic algorithms due to constraints and composition difference of this scheme.

\section{Cloud Optimization Strategy}

The nodes of cloud layer are taking the smart power distribution system or idle computing resources in power grid enterprise as the foundation platform. It is composed of a variety of levels, for example, smart distribution network has low pressure, medium pressure and high pressure levels. So, it uses the breadth first heuristic algorithm for the optimization in cloud layer. The steps of this algorithm are as follows:

- By visiting vertex $V_{0}$. It is a stochastic selected node from non-vector graph $G$, namely a vertex $V_{0}$

- Those neighbor nodes of $V_{0}$ are the set of the search node, namely as $\left\{V_{1}, V_{2}, \ldots, V_{k}\right\}$, which are connected to each other and have not visited. Then, starting from $V_{l}$ in the search node set, the weight $W_{i}$ of each node $V_{i}$ to node $V_{0}$ is obtained and form the weights set $\left\{W_{1}, W_{2}, \ldots, W_{k}\right\}$. Next, it is sequentially accessed from each unvisited neighbor node $V_{i}$ and forms different the weight set

- Repeat step 2) until all nodes has been visited. The weight of any two nodes is gain according to the weight set in arbitrary nodes. When the weight is ascending, the best route selection is the minimum weight

\section{End Optimization Strategy}

The computing nodes of end layer are connected by communities, enterprises, buildings, residents and so on, according to the principle of regional division. Most of them are radial structure, so the depth first heuristic algorithm is chosen to the optimization. The steps of this algorithm are as follows:

- A node $V_{i}$ of the non-vector graph $G$ is selected as the vertex and the starting point. Then, the vertex is visited and labeled 
- The node $V_{i}$ is the current vertex, the node $V_{j}$ of each adjacent $V_{i}$ is searched by recording the weight $W_{i j}$ of node $V_{i}$ to node $V_{j}$. If $V_{j}$ is not visited, the neighbor node $V_{j}$ will be accessed and marked. If the node $V_{j}$ has been visited, it will search for the next neighbor node of $V_{i}$

- The node $V_{j}$ is the current vertex, repeat step 2). When in the non-vector graph $G$ all nodes have been visited, which has the path connected vertex to the node $V_{i}$

- If the vertex is non-connected vertex in the nonvector graph $G$, an unvisited vertex is selected to as the starting point. Then repeat the above process until all vertices are visited

- According to the weight distribution, the weight of any two nodes can be calculated. When the weight is ascending order arrangement, the best routing selection is the first weight

\section{Economic Model of Construction of Cloud + end in DSM}

By the virtual external IT pool, collaborative innovation, resource integration, cloud + end integrated solutions could solve the technical problem of multisource heterogeneous, multi user attributes in DSM, which completely change the traditional IT processing mode. Specifically, it changes the record system to adhesion system, the IT management mode and the internal business needs to customer oriented model. It also considers the DSM business model, the feasibility of the operation and maintenance of the rationality and other practical issues.

However, the construction of the DSM cloud + end is related to many stakeholders, such as power grid enterprise, government, equipment suppliers and terminal users. How to organize and coordinate those stakeholders is an arduous task. Also, it involves many economic problems, such as large scale and wide implementation of the project, the policies of the energy-saving emission reduction and energy costs of mass users and so on. It is difficult to balance the interests from multi members in the construction of the DSM. An economic model is essential to the dynamic economic problem of the stakeholders in DSM based on the game theory.

\section{Game Theory}

According to the form of the game, the game theory can be divided into three forms, non-cooperative game, cooperative game and evolutionary game. Generally speaking, the game theory is composed of three parts, means participants, strategies and payments. Namely $G$ $=\left\{N ; S_{1}, S_{2}, \ldots, S_{n} ; u_{1}, u_{2}, \ldots, u_{n}\right\}$ :

$$
G=\left\{N ; S_{1}, S_{2}, \ldots, S_{n} ; u_{1}, u_{2}, \ldots, u_{n}\right\}
$$

where, the participants are $N=\{1,2, \ldots, n\}, S=\left\{S_{1}, S_{2}, \ldots\right.$, $\left.S_{n}\right\}$ represents a strategy set for all participants, $S_{i}=\left\{s_{1}\right.$, $\left.s_{2}, \ldots, s_{n}\right\}$ is the strategy set of the $i$-th participant, $u$ $=\left\{u_{1}, u_{2}, \ldots, u_{n}\right\}$ is a set and represents the payment vector of all participants, $u_{i}$ is the payment of the $i$-th participant in game.

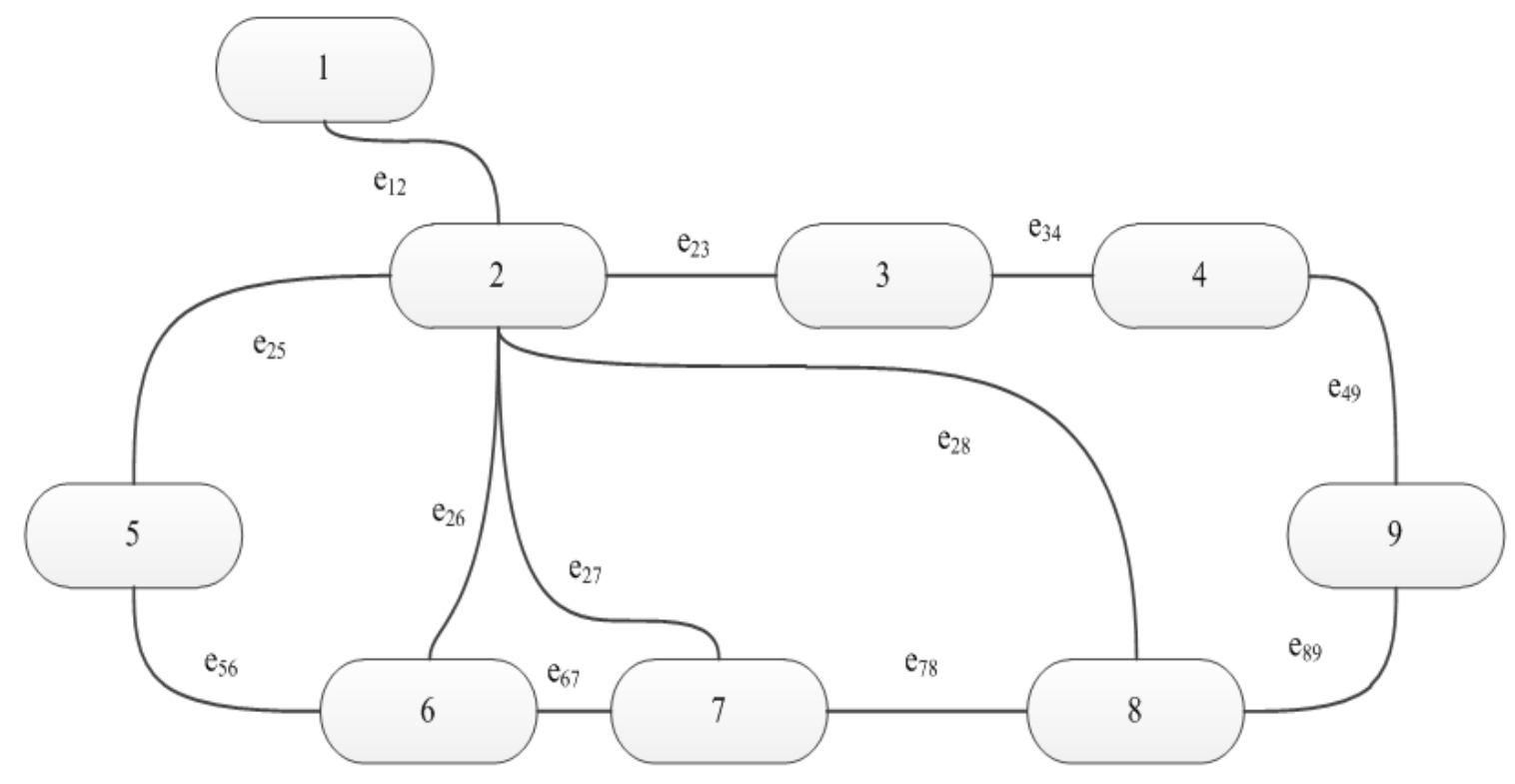

Fig. 7. Route optimization strategy model of cloud + end 


\section{Mathematical Model}

In order to balance the interests of large-scale participants in DSM, the construction model is a cooperative game in this study.

\section{Game Participants}

The participants of the construction in DSM include power grid enterprises, government and the third party organizations, namely $A, B, C$. The third party organizations can be involved to remove $A, B$ in the general overview.

\section{Game Strategy}

When the game theory are built among $A, B$ and $C$, the strategy is a variety of forms of capital from $\mathrm{A}, B$ and $\mathrm{C}$, such as cash, technology, resources, policies, etc.. It is recorded as $M_{A}, M_{B}, M_{C}$ for those participants. The capital of each participant is a continuous range, namely $S_{A}, S_{B}, S_{C}$. So, the game strategy formulates in (4):

$$
\left\{\begin{array}{l}
M_{A} \in\left\{S_{A}=\left[\mathrm{M}_{A}^{\min }, \mathrm{M}_{A}^{\max }\right]\right\} \\
M_{B} \in\left\{S_{B}=\left[\mathrm{M}_{B}^{\min }, \mathrm{M}_{B}^{\max }\right]\right\} \\
M_{C} \in\left\{S_{C}=\left[\mathrm{M}_{C}^{\min }, \mathrm{M}_{C}^{\max }\right]\right\}
\end{array}\right.
$$

\section{Payment Vector}

The payment vector of $A, B, C$ is a price difference between the investment income and the investment cost of the construction, namely $U_{A}, U_{B}, U_{C}$. The investment income of all the participants are mainly energy savings $U_{e}$, labor costs savings $U_{p}$, energy saving and emission reduction $U_{d}$, etc. On the other hand, the investment costs of all participants are the bank's loan interest includes $M_{A}, M_{B}$ and $M_{C}$. Therefore, a payment vector of the $i$-th participant can be expressed as follow:

$$
U_{i}=U_{i e}+U_{i p}+U_{i d}-U_{i f}-M_{i A}-M_{i B}-M_{i C}
$$

\section{Nash Equilibrium}

Since the participants of the construction in DSM are considered as a whole, it can be defined as a cooperative game model. Then the Nash equilibrium point is defined in formulation (6):

$$
\left(\mathrm{M}_{A}^{* \prime}, \mathrm{M}_{B}^{* \prime}, \mathrm{M}_{C}^{* \prime}\right)=\arg \max _{M_{A}, M_{B}, \mathrm{M}_{C}} U_{A B C}\left(\mathrm{M}_{A}^{* \prime}, \mathrm{M}_{B}^{* \prime}, \mathrm{M}_{C}^{* \prime}\right)
$$

where, $\left(\mathrm{M}_{A}^{* \prime}, \mathrm{M}_{B}^{* \prime}, \mathrm{M}_{C}^{* \prime}\right)$ is a Nash equilibrium point and $\mathrm{U}_{A B C}\left(\mathrm{M}_{A}^{* \prime}, \mathrm{M}_{B}^{* \prime}, \mathrm{M}_{C}^{* \prime}\right)$ is a cooperation strategy for $A, B$ and $C$.

The solution for the game model is a complex process and it has many methods. According to the Nash equilibrium theory, we can have many algorithms to solve the optimization strategy based on the concrete situation in DSM, including ant algorithm, neural network algorithm, genetic algorithm and so on.

\section{Practical Application}

A city in southern China is chosen for testing the proposed method. It has the most industrial electricity consumption and the second most whole social demand of electric power in China. The Chinese government puts forward the policy of the upper limit of the total energy consumption in city in 2013 . To reduce the total energy consumption in this city, a "1-3-3"object is proposed to implement 1000 MW electric load decrease and 3000, $000,000 \mathrm{kWh}$ annual electricity consumption decrease. If the goals can been achieved, 3000 companies and a large number of resident users are incorporated into the DSM. Furthermore, it is necessary to build a DSM cloud + end public service system.

\section{Architecture of DSM Platform}

The system architecture of the public service cloud for DSM in southern China is the cloud + end, the detail relationship is shown in the Fig. 8. The main function of this platform is focused on intelligent residential power distribution automatic demand response, mutual power, optimization operation modes of end users, efficiency improvement of power system, industry electricity standards development, etc[].

The city's DSM cloud service platform includes two private clouds, which are government cloud and the power grid enterprise cloud.

The government cloud is responsible for statistical analysis of the city's DSM data, providing data reference for the policies of DSM. It announces the DSM result linking with the portal website. At the same time, the government cloud is responsible for transmitting the interactive information to the end layer servers, which belongs to industry, business, resident and other users.

The power grid enterprise cloud is responsible for the collection and monitoring of all systems in the end layer of DSM, using big data analysis theory for the timely response strategy based on different user requirements,. On the other hand, it support the DSM through cloud computing, such as the integration of the idle resource in power grid enterprise, the optimization, control and operation of power system, online monitoring of users' overall equipment, orderly use of electricity, cutting peak load and so on.

The end server is an information collection and key points of the cloud + end integration solution. According to different configurations and attributes in user, it is divided to four aspects of industrial, business, residential and the third party organization users. Its main functions are the communication protocol analysis, the information 
collection for users and data transmission to the cloud platform. At the same time, it receives the respond command from clouds and parses the command to send the different equipment in DSM users, so that all users could be involved in DSM through mobile phone, iPad, Internet, etc[]..

\section{Construction Model}

By coordinating power grid enterprise (A), government (B) and the third party organizations (C), a joint owned construction mode is selected to build the city DSM cloud public service platform. Their game strategy and payment vector are shown in Table 2 and 3.

The construction model is composed of a government guide, a power grid enterprise agencies and the third part organization monitor, invested respectively 10000000 , 1000000,3000000 RMB. In additional, the government is responsible for the procurement of the DSM and the management of the relevant policies and subsidies. The power grid enterprise constructs the DSM platform through the bill of user. The third part organization assesses the implementation of the project through the evaluation index.
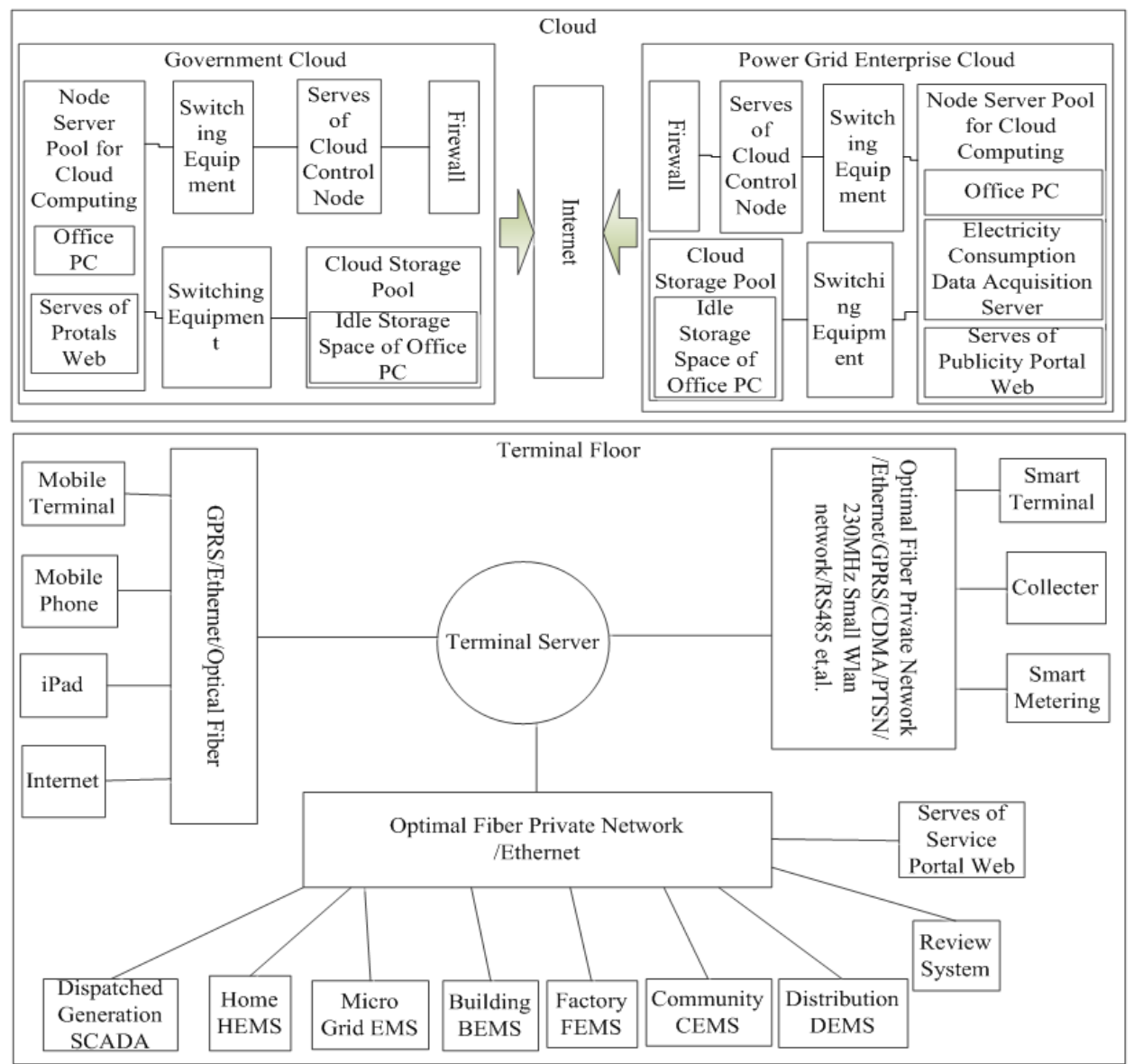

Fig. 8. Architecture diagram of DSM cloud + end

Table 2. The game strategy

\begin{tabular}{|c|c|c|c|c|c|}
\hline No. & Cash (RMB) & Technology (RMB) & Resource (RMB) & Policy (RMB) & Total(RMB) \\
\hline A & 5000000 & 3000000 & 2000000 & & 10000000 \\
\hline B & & & & 1000000 & 1000000 \\
\hline $\mathrm{C}$ & & 2000000 & 1000000 & & 3000000 \\
\hline
\end{tabular}

Table 3. The payment vector

\begin{tabular}{llll}
\hline No. & Annual income (RMB) & Annual cost (RMB) & Recycle period (Year) \\
\hline A & 2000000 & 700000 & 5.8 \\
B & 1000000 & 70000 & 1.1 \\
C & 1500000 & 210000 & 2.4 \\
\hline
\end{tabular}




\section{Discussion}

For the purposes of this paper, it is necessary only to suppose that DSM and cloud computing can be widely accepted. DSM is increasingly playing an important role in Smart Grid and has some difficult problems of a wide range of different needs of the user group. Cloud computing provides a better solution for these problems. However, the construction of DSM based on cloud computing has a large number of participants, which is difficult to manage. In this study, it presents a scheme design of two aspects of technology and economy:

- It proposes the technical scheme design of cloud+ end technology. End can be better to solve the standardized access of large scale users. Cloud provides a solid foundation for the application of DSM

- The construction model based on game theory can resolve the problem of numerous participants, which want to get their best interests

- The actual demonstration project construction in China verifies the feasibility of the scheme design and is only a case. More successful promotion needs more cases to demonstrate the application of the scheme design in the world

\section{Conclusion}

This paper studies the feasibility of applying cloud computing technology into the DSM for the technical problems and discusses the rationality of the game theory for the economic problems in the construction process of the DSM. A demonstration project is used to test the performance of the proposed method in a southern city of China. The results demonstrate that the DSM could be efficiently managed by the cloud + end method.

However, some practical problems should be further studied about DSM, such as personal information security, policy adaptability and equipment property.

\section{Acknowledgement}

The authors gratefully acknowledge support provided by the National Natural Science Foundation of China (NO.51377115) and the National High Technology Research and Development Program of China (NO. 2014AA052003).

\section{Funding Information}

The authors acknowledge the funders of this manuscript. The title of NO.51377115 is a fast mode and simulation of smart distribution system based cloud computing.

\section{Author's Contributions}

The authors state the contributions made by each author in the preparation, development and publication of this manuscript.

\section{Ethics}

The authors address any ethical issues that may arise after the publication of this manuscript.

\section{References}

Adika, C.O. and L. Wang, 2014. Smart charging and appliance scheduling approaches to demand side management. Int. J. Elect. Power Energy Syst., 57: 232-240. DOI: 10.1016/j.ijepes.2013.12.004

Alireza, Z., S. Jadid and P. Siano, 2014. Smart microgrid energy and reserve scheduling with demand response using stochastic optimization. Int. J. Elect. Power Energy Syst., 63: 523-533.

DOI: $10.1016 / j$.ijepes.2014.06.037

Assunção, M.D., R.N. Calheiros, S. Bianchi, M.A.S. Netto and R. Buyya, 2015. Big Data Computing and Clouds: Trends and Future Directions. J. Parallel Distrib. Comput., 79: 3-15.

DOI: $10.1016 /$ j.jpdc.2014.08.003

Blake, M.B., 2007. Decomposing composition: Serviceoriented software engineers. IEEE Software, 24: 68-77. DOI: 10.1109/MS.2007.162

González-Martínez, J.A., M.L. Bote-Lorenzo, E. GómezSánchez and R. Cano-Parra, 2015. Cloud computing and education: A state-of-the-art survey. Comput. Educ., 80: 132-151.

DOI: 10.1016/j.compedu.2014.08.017

Ji, P., X. Ma and G. Li, 2015. Developing green purchasing relationships for the manufacturing industry: An evolutionary game theory perspective. Int. J. Product. Econom., 166: 155-162. DOI: 10.1016/j.ijpe.2014.10.009

Li, D., J. Ma, Z. Tian and H. Zhu, 2015. An evolutionary game for the diffusion of rumor in complex networks. Phys. A, 433: 51-58. DOI: 10.1016/j.physa.2015.03.080

Logenthiran, T., D. Srinivasan and T.Z. Shun, 2012. Demand side management in smart grid using heuristic optimization. IEEE Trans. Smart Grid, 3: 1244-1252. DOI: 10.1109/TSG.2012.2195686

Lopez, M.A., S. de la Torre, S. Martín and J.A. Aguado, 2015. Demand-side management in smart grid operation considering electric vehicles load shifting and vehicle-to-grid support. Int. J. Elect. Power Energy Syst., 64: 689-698.

DOI: $10.1016 /$ j.ijepes.2014.07.065 
Malik, A.S., 2001. Modelling and economic analysis of DSM programs in generation planning. Int. J. Elect. Power Energy Syst., 23: 413-419. DOI: 10.1016/S0142-0615(00)00077-6

Sheikhi, A., M. Rayati, S. Bahrami, A.M. Ranjbar and S. Sattari, 2015. A cloud computing framework on demand side management game in smart energy hubs. Int. J. Elect. Power Energy Syst., 64: 1007-1016. DOI: 10.1016/j.ijepes.2014.08.020
Vasile, A., C.E. Costea and T.G. Viciu, 2012. An evolutionary game theory approach to market competition and cooperation. Adv. Complex Syst., 15: 1-15. DOI: 10.1142/S0219525912500440

Wang, W., P. Wang, G. Xiao and S. Gong, 2014. Power demand and supply management in microgrids with uncertainties of renewable energies. Int. J. Elect. Power Energy Syst., 63: 260-269.

DOI: 10.1016/j.ijepes.2014.05.067 